\title{
Internal herniation of sigmoid colon into ladd's band
}

\author{
C.R. Praveen ${ }^{1}$, B.N. Patowary ${ }^{2}$, S Kumar ${ }^{1}$,P Shrestha ${ }^{3}$ \\ ${ }^{1}$ Lecturer, ${ }^{2}$ Professor, ${ }^{3}$ Resident, Department of General Surgery, College of Medical Sciences, Bharatpur, Chitwan, Nepal.
}

\begin{abstract}
Ladd's bands are generally known to cause duodenal and small bowel obstruction. Sigmoid colon compression by Ladd's bands has not been reported till now though a few cases of colonic obstruction have been reported. We report a case of sigmoid colon obstruction secondary to its herniation into Ladd's band in a child with intestinal malrotation.
\end{abstract}

Key Words: Ladd's band, sigmoid colon, herniation, intestinal malrotation.

\section{Introduction}

Ladd's bands are secondary to incomplete intestinal rotation during fetal development and represent continuous growth of peritoneal folds fixing the duodenum and cecum to the retroperitoneum. ${ }^{1}$ Though it is commonly known to cause duodenal obstruction, it rarely causes colonic obstruction with only a few reported cases till now. These cases including ours show that intestinal malrotation and Ladd's band need to be kept in mind while treating a case of acute and chronic abdominal pain in older children.

\section{Case report}

A 12-year-old boy presented with complaints of pain lower abdomen for 10 days which had increased since two days with intermittent episodes of non-bilious vomiting. He had been treated elsewhere conservatively for two days before being referred to

Correspondence: C.R. Praveen

Email:praveencsr@hotmail.com our hospital. He had no history of constipation or obstipation. However, he did complain of intermittent episodes of lower abdominal distension appearing hours after food intake, which was relieved after passing stools with difficulty. There was no bleeding per rectum. On abdominal examination he had tenderness and rebound tenderness around the umbilicus and right iliac fossa, with guarding and mild distension of lower half of abdomen. However, there was no evidence of free fluid.

Bowel sounds were sluggish. Per rectal examination was normal. Erect view $\mathrm{x}$-ray of the abdomen showed no evidence of air fluid levels or free air under the diaphragm. Ultrasonography of the abdomen revealed no significant findings, except minimal free fluid in the peritoneal cavity in right iliac fossa and pelvis. Exploratory laparotomy was done in view of the abdominal findings suggestive of peritonitis. On exploration intestinal malrotation was detected. A loop of sigmoid colon had herniated into a loop formed by Ladd's band, causing incomplete luminal compression. 
Journal of College of Medical Sciences-Nepal,2011,Vol-7,No-3 The proximal colon was mildly dilated; however, there were no features of ischemia or gangrene. This loop of sigmoid colon was in the right iliac fossa and was viable with no features of ischemia. There was minimal free clear serous fluid in the right iliac fossa and pelvis.

The Ladd's band was found arising below the region of duodenum, which was normal, crossed the spine, and had no features of compression. This band continued down to form a loop into which a segment of sigmoid colon was found internally herniated. The band was found to end near the cecum, which was in the right hypochondrium and was not fixed to the retroperitoneum. The small bowel distal to duodenojejunal flexure was found inside a primitive hernial sac and there was no volvulus or shortening of small bowel mesentery. Ladd's band was divided and reduction of the internal herniation was done. Appendicectomy was done followed by cecopexy. Post operative period was uneventful and there were no symptoms of pain abdomen and obstruction in follow up visits on first and fifth week.
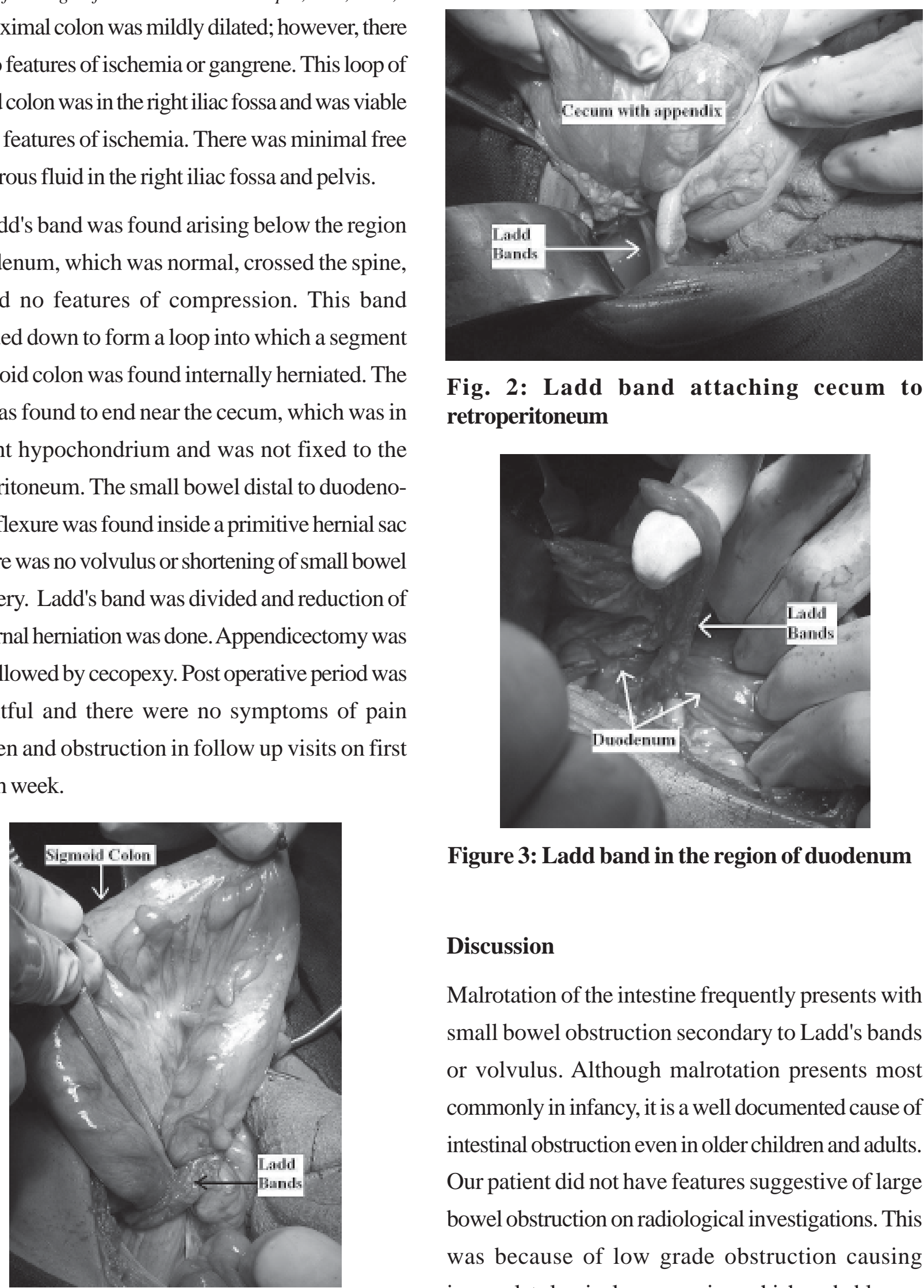

Fig. 2: Ladd band attaching cecum to retroperitoneum

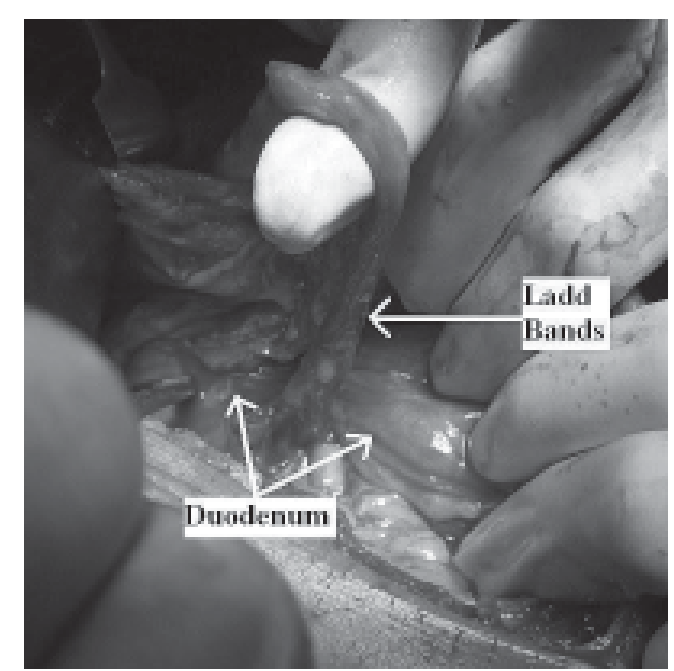

Figure 3: Ladd band in the region of duodenum

\section{Discussion}

Malrotation of the intestine frequently presents with small bowel obstruction secondary to Ladd's bands or volvulus. Although malrotation presents most commonly in infancy, it is a well documented cause of intestinal obstruction even in older children and adults. Our patient did not have features suggestive of large bowel obstruction on radiological investigations. This was because of low grade obstruction causing incomplete luminal compression, which probably was the reason for intermittent episodes of pain and 
C.R. Praveen et al.Internal herniation of sigmoid colon into ladd bands

vomiting. This internal herniation of sigmoid colon was recent, explaining the onset of symptoms at this age.

Till now few cases of colonic obstruction associated with intestinal malrotation have been reported. In one case, the patient had a nonfixated colon with normal rotation, and the obstruction was caused by an abnormal vascular arcade at the level of the descending colon. ${ }^{2}$ In another case, an infant developed colonic obstruction after a knuckle of transverse colon was caught in a classic midgut volvulus. ${ }^{3}$ Complete obstruction of the hepatic flexure of the colon secondary to a Ladd's band in a previously healthy teenage boy has also been reported. ${ }^{4}$

Ladd's bands causing problems as late as 65 years has been reported. This was a case of a 65 -year- old male in whom it was the cause of small bowel obstruction and strangulation. ${ }^{5}$ Malrotation in older children and adults may present acutely with obstruction or volvulus, with chronic abdominal pain, or incidentally during diagnostic tests for other abdominal pathology. It is likely that atypical presentations of malrotation are more common in the older children and adults. ${ }^{6}$

Thus a high index of suspicion is required in these cases especially those who present with chronic abdominal pain. In our case the patient had been treated elsewhere conservatively before being referred to our hospital. Fortunately our patient did not have bowel ischemia or gangrene as the obstruction was incomplete with no vascular compromise. Because of these dire consequences we need to be aware of intestinal malrotation and consider it in our differential diagnosis even in a patient with chronic abdominal pain.

\section{References}

1. D.J. Hackman, T.C. Grikscheit, K.S. Wang, et.al. Paediatric Surgery. F.C. Brunicardi, D.K. Andersen, J.G. Hunter, et.al. Schwartz's Principles of Surgery. 9 $^{\text {th }}$ ed. New York: Mc Graw Hill; 2010: 1428-9.

2. J Jansen, C Driver, J Duncan. Unusual hindgut malrotation and associated mesenteric vascular abnormality presenting as large bowel obstruction in an adult. Surgeon 2007;5:109-10.

3. P Parag, J Chacko, G Thomas. Colonic obstruction in classical malrotation. Indian Pediatr 2000;37:440-1.

4. T Raphaeli, C Parimi, K Mattix, P.J. Javid. Acute colonic obstruction from Ladd bands: a unique complication from intestinal malrotation. J Pediatr Surg. 2010 Mar; 45:630-1

5. M Mongardini, C.M. Moschella , F Schillaci, et.al. Strangulated abdominal herniation by Ladd's band. $G$ Chir. 2005 May; 26:207-9.

6. F.M. Von, U Herzog, C Ackerman, et.al. Acute and chronic presentation of intestinal nonrotation in adults. Dis Colon Rectum 1994; 37:192-8. 